\title{
Atmospheric and oceanic excitation of the rotation of a three-layer Earth
}

\author{
V. Dehant ${ }^{1}$, O. de Viron ${ }^{1}$, and M. Greff-Lefftz ${ }^{2}$ \\ 1 Royal Observatory of Belgium, Avenue Circulaire 3, 1180 Brussels, Belgium \\ e-mail: v.dehant@oma.be \\ ${ }^{2}$ Institut de Physique du Globe, Paris, France
}

Received 19 October 2004 / Accepted 24 February 2005

\begin{abstract}
In this paper, we evaluate the nutational Earth response to the excitation exerted by a surface fluid (atmosphere, ocean, and hydrology) for a simple Earth model, constituted of three homogeneous layers: a solid deformable inner-core, a liquid outer core, and an elastic mantle. Our formula, valid in the quasi-diurnal frequency band, includes two resonances, at the Free Core Nutation (FCN) and the Free Inner-Core Nutation (FICN). Additionally, we have evaluated the amplitudes of those wobbles in response to a random noise excitation. We show that, compared with the FCN signal, the resonance at the FICN frequency induced by a surface fluid layer only induces a very small signal in the Earth rotation, and that, with an excitation comparable to the one available at the FCN, the FICN would generate a signal at the Earth surface at the sub-micrometer level.
\end{abstract}

Key words. Earth - reference systems

\section{Introduction}

The variable Earth rotation is classically separated into three motions: (i) the motion of the mean Earth rotation axis in space, i.e. the precession-nutation; (ii) the motion of the mean Earth around its rotation axis, called polar motion (PM); and (iii) the variation of the Earth rotation velocity, studied in terms of the variation of the length-of-day (LOD). The variations of the length-of-day are mostly excited by the atmosphere (more than $95 \%$ at periods ranging from a few days to several years); the polar motion is excited by the ocean and the atmosphere at the same level; and the precession-nutation mainly corresponds to the response of the Earth to the gravitational action of the Sun, the Moon, and the other planets.

Nutation is observed using Very Long Baseline Interferometry (VLBI) with a precision better than a milliarcsecond (mas) for any observing session, corresponding to the sub-centimeter level at the Earth's surface. The error on a given harmonic nutation coefficient is even better. For most of the nutation waves, the periods are imposed by the periods of the external forcing, and the internal structure only affects the magnitude of the response. In addition, the presence of a fluid core and a solid inner core allows the existence of two normal modes of which the periods are determined by the physical characteristics of the Earth. The nutations are excited by the gravitational forcing from the Sun, the Moon and the other planets, as well as by the geophysical fluids. The atmosphere and ocean indeed influence the nutations at those frequencies as well, but the effects are small relative to the Sun and the Moon gravitational forcing. As no external wave corresponds exactly to the periods of the free modes, only the geophysical fluids can excite the free nutation modes (with a possible exception related to the excitation by earthquakes). In particular, the atmosphere and the ocean are thought to be the major excitation sources of the Free Core Nutation (FCN), see Sasao \& Wahr (1981). The Free Inner-Core Nutation (FICN) free mode is observed by VLBI at the level of a few tenths of mas (see Herring et al. 1991, 2002). Its amplitude varies with time. In addition the presence of those free modes amplifies the Earth response for the waves of frequency close to the resonance frequencies.

The nutation motion is modeled, based on celestial mechanics on the one hand, and on physics of the Earth interior on the other hand. The nutation model adopted by the International Astronomical Union and the International Union of Geodesy and Geophysics (see Mathews et al. 2002) (MHB2000) considers time varying amplitude of the FCN free mode. The FICN free mode was not considered. Both modes are believed to be excited by the atmosphere and the ocean.

The nutational response of the Earth to the gravitational forcing is classically expressed in terms of a transfer function (TF), so that, for any frequency $\sigma$ of the forcing potential, the nutation response is given by

Nutation of real $\operatorname{Earth}(\sigma)=T F(\sigma) \cdot$ Nutation for rigid $\operatorname{Earth}(\sigma)$ 
Table 1. Mean value and interval of values for the periods and quality factors of the FCN and FICN.

\begin{tabular}{l|ll}
\hline \hline & Mean value & Interval \\
\hline FCN period (in days) & 430.2 & {$[430.0,430.4]$} \\
FCN Q & 20000 & {$[19000,21500]$} \\
FICN period (in days) & 1030 & {$[840,1310]$} \\
FICN Q & 680 & {$[520,1020]$} \\
\hline
\end{tabular}

where the nutations for the rigid Earth are computed directly from the celestial mechanics. The fluid effects, i.e. the tidal (and nontidal) ocean and the atmospheric effects, are added separately, as they are not proportional to and in phase with the gravitational potential. In the adopted model, as the atmospheric and oceanic forcing are not known as precisely as the external forcing, their effects on the classical nutation waves are fit (in the atmosphere case) or scaled (ocean case) on the observed nutation amplitudes.

Up to now, the transfer function representing the response of a three-layer Earth to this excitation has never been evaluated. The amplitude of the inner-core mode is thought to be small, as the inner-core inertia only accounts for one thousandth of the total Earth inertia. Nevertheless, the observations become increasingly accurate, and the inner core mode signature might soon reach the observational level. As this signature depends on the internal structure of the Earth, its observation would lead to a better understanding of the Earth's deep interior.

The IAU MHB2000 model of Mathews et al. (2002) is based on the fit of the parameters of a simple Earth model on the nutation observations. The associated transfer function includes both FCN and FICN resonance in the Earth response to gravitational forcing. In that study, the period and damping of these modes are estimated from the observed VLBI nutations; the periods are 430 and 1030 days for the FCN and FICN respectively and their damping factors, represented by the so-called quality factor, defined as the ratio between the real and the imaginary part of the frequency divided by two, are 20000 and 680 respectively. Dehant et al. (2003) have shown that any value within the intervals given in Table 1 is acceptable as well, considering the uncertainties on the determinations of nutation amplitudes.

Observation precision gets better with time, due to the improvements in technology and in observation strategy. When the modelled nutation (MHB2000 without the free modes) is subtracted from the observation, the signal associated with the free core nutation is the major source of residuals. From the analysis of those residuals, Feissel-Vernier (2003) and Feissel-Vernier et al. (2005) have shown the presence of some power in the FICN frequency band. This motivated us to compute theoretically the nutational response of a three-layer Earth for the normal nutation modes excited by the noise of the atmosphere and/or the ocean at those frequencies.

In addition to the nutation, the Earth response to the gravitational and geophysical fluid forcing also includes deformations of its different layers. In our first order approach, the effects of the deformations are assumed to be linear in the potential. The proportionality coefficients between the forcing potential (or surface/internal pressure/load distribution) and the deformation are called Love numbers.

The paper is organized as follows: Sect. 2 describes the model used and the major hypotheses done in our study, Sect. 3 is devoted to the theoretical computation of the Earth response to atmospheric/oceanic forcing; Sect. 4 gives the free mode periods and the transfer functions for the diurnal wobbles associated with nutations. Section 5 is devoted to the computation of the Love numbers (i.e. transfer functions for deformation) involved and the numerical values of the excitation function, and Sect. 6 provides the Earth response at the FICN period to random noise atmospheric/oceanic excitation. Conclusions and discussion are given in Sect. 7.

\section{Model used and main hypotheses}

In our study, we used a simplified Earth model, composed of three homogeneous ellipsoidal layers, an elastic inner-core, a fluid core, and an elastic mantle. The three layers are assumed to only move "rigidly": small rotations are allowed with respect to the mean diurnal rotation. Additionally, deformations are allowed, which are accounted for by using the Love number formalism. The coupling between the fluid and solid parts is assumed to be only due to the pressure acting on the ellipsoidal boundaries and to the gravitational interaction between the three layers. Nevertheless, considering only non-dissipative forces would lead to infinite amplitude of the free modes, which is unphysical. Consequently, we add to the frequency of the mode an imaginary part which accounts for the dissipation. This dissipation would come from viscosity in the Earth, topographic coupling at the core boundaries, and electromagnetic coupling between the core and the solid layers. The surface fluid layer is assumed to be "thin layer". The excitation exerted by the atmosphere and the ocean on the Earth nutation is assumed to be a white noise in a given frequency band but some particular frequencies are identified as relevant for the atmosphere or ocean dynamics. As the angular momentum approach is used for computing the effect of the fluid layer, we do not need to assess any properties of the interaction between the surface fluid layer and the mantle. 


\section{Theoretical excitation of the free modes}

The rotation dynamic of a simple Earth model is classically analyzed using the Liouville equation, which is a particular case of the angular momentum budget equation. We used a reference frame rotating with the mantle, with a rotation vector $\mathbf{\Omega}$, defined by

$\boldsymbol{\Omega}=\Omega\left(\begin{array}{c}m_{1} \\ m_{2} \\ 1+m_{3}\end{array}\right)$

where $\Omega$ is the Earth mean angular velocity. Note that the rotation perturbation $m_{i}$ are small with respect to 1 .

The inertia of the whole Earth, $\underline{\bar{I}}$, is the one of a biaxial ellipsoid perturbed by small deformations:

$\underline{I}=\left(\begin{array}{ccc}A & 0 & 0 \\ 0 & A & 0 \\ 0 & 0 & C\end{array}\right)+\bar{c}$.

The incremental part $\underline{\bar{c}}$ is due to the deformation of each layer in response to the forcing potential, as well as to the change in the atmospheric and oceanic inertia. All the $c_{i j}$ are small with respect to the equilibrium moment of inertia.

The angular momentum of the whole Earth is then given by

$\boldsymbol{H}=\underline{\bar{I}} \mathbf{\Omega}+\boldsymbol{h}$.

The addititional angular momentum $\boldsymbol{h}$ is associated with the relative angular momentum of the different layers of the whole Earth: the inner core $\left(\boldsymbol{h}_{\mathrm{s}}\right)$, the fluid outer core $\left(\boldsymbol{h}_{\mathrm{f}}\right)$, and the atmosphere $\left(\boldsymbol{h}_{\mathrm{a}}\right)$. Those additional angular momenta are also small quantities.

As explained in Sect. 2, we only allow rigid rotation of the core and inner core. Consequently, we have:

$\boldsymbol{h}_{\mathrm{s}}=\bar{I}_{\mathrm{s}} \omega_{\mathrm{s}}$

$\boldsymbol{h}_{\mathrm{f}}=\overline{\bar{I}}_{\mathrm{f}} \omega_{\mathrm{f}}$.

The inertia of the inner core and outer core are given by

$\underline{\bar{I}_{\mathrm{s}}}=\left(\begin{array}{ccc}A_{\mathrm{s}} & 0 & 0 \\ 0 & A_{\mathrm{s}} & 0 \\ 0 & 0 & C_{\mathrm{s}}\end{array}\right)+\bar{c}_{\mathrm{s}}$

$\underline{\bar{I}_{\mathrm{f}}}=\left(\begin{array}{ccc}A_{\mathrm{f}} & 0 & 0 \\ 0 & A_{\mathrm{f}} & 0 \\ 0 & 0 & C_{\mathrm{f}}\end{array}\right)+\underline{\bar{c}_{\mathrm{f}}}$.

With respect to the mantle, the rotation vectors of the inner core and outer core are

$\omega_{\mathrm{s}}=\Omega\left(\begin{array}{l}m_{1}^{\mathrm{s}} \\ m_{2}^{\mathrm{s}} \\ m_{3}^{\mathrm{s}}\end{array}\right)$
$\omega_{\mathrm{f}}=\Omega\left(\begin{array}{l}m_{1}^{\mathrm{f}} \\ m_{2}^{\mathrm{f}} \\ m_{3}^{\mathrm{f}}\end{array}\right)$.

As for the solid Earth, the quantities $\bar{c}_{\mathrm{s}}, \bar{c}_{\mathrm{f}}, m_{\mathrm{i}}^{\mathrm{f}}$, and $m_{\mathrm{i}}^{\mathrm{s}}$ are small. The time derivative, in the inertial frame, of the angular momentum for the solid Earth is given by

$\frac{\mathrm{d} \boldsymbol{H}}{\mathrm{d} t}=\frac{\partial \boldsymbol{H}}{\partial t}+\mathbf{\Omega} \wedge \boldsymbol{H}$

Using the expression developed here above, it is straight forward to get the equatorial part of the Liouville equations, at the first order in the small quantities, for the whole Earth as:

$A \dot{m}-\mathrm{i} \alpha A \Omega m+\dot{c}+\mathrm{i} \Omega c+A_{\mathrm{f}} \dot{m}_{\mathrm{f}}+i \Omega A_{\mathrm{f}} m_{\mathrm{f}}+A_{\mathrm{s}} \dot{m}_{\mathrm{s}}+\mathrm{i} \Omega A_{\mathrm{s}} m_{\mathrm{s}}+h_{\mathrm{a}}=\frac{\Gamma}{\Omega}$

where $\alpha=(C-A) / A$ is the dynamic flattening of the whole Earth and $\Gamma$ the total torque acting on the Earth. Similarly, we will note $\alpha_{\mathrm{f}}=\left(C_{\mathrm{f}}-A_{\mathrm{f}}\right) / A_{\mathrm{f}}$ and $\alpha_{\mathrm{s}}=\left(C_{\mathrm{s}}-A_{\mathrm{s}}\right) / A_{\mathrm{s}}$. 
The $x$ and $y$ components have been complexely added, so that $f=f_{1}+\mathrm{i} f_{2}$. In particular, the complex wobble are defined as

$$
\begin{aligned}
& m=m_{1}+\mathrm{i} m_{2} \\
& m_{\mathrm{f}}=m_{1}^{\mathrm{f}}+\mathrm{i} m_{2}^{\mathrm{f}} \\
& m_{\mathrm{s}}=m_{1}^{\mathrm{s}}+i m_{2}^{\mathrm{s}} \\
& c=c_{13}+\mathrm{i} c_{23} \\
& c_{\mathrm{f}}=c_{\mathrm{f}, 13}+\mathrm{i} c_{\mathrm{f}, 23} \\
& c_{\mathrm{s}}=c_{\mathrm{s}, 13}+\mathrm{i} c_{\mathrm{s}, 23} .
\end{aligned}
$$

The rotation vector of the inner core and the outer core in the inertial frame is given by

$\mathbf{\Omega}_{\mathrm{s}}=\mathbf{\Omega}+\omega_{\mathrm{s}}$

$\mathbf{\Omega}_{\mathrm{f}}=\mathbf{\Omega}+\omega_{\mathrm{f}}$.

In the case of those two layers, there is no differential angular momentum, as we consider they are rotating rigidely. Consequently, the angular momentum budget equation, at the first order in the small quantities, are given by:

$A_{\mathrm{s}} \dot{m}+A_{\mathrm{s}} \dot{m}_{\mathrm{s}}-\mathrm{i} \Omega \alpha_{\mathrm{s}} A_{\mathrm{s}} m+\dot{c}_{\mathrm{s}}+\mathrm{i} \Omega c_{\mathrm{s}}+\mathrm{i} \Omega A_{\mathrm{s}} m_{\mathrm{s}}=\frac{\Gamma_{\mathrm{s}}}{\Omega}$

for the inner core, and

$A_{\mathrm{f}} \dot{m}+A_{\mathrm{f}} \dot{m}_{\mathrm{f}}-\mathrm{i} \Omega \alpha_{\mathrm{f}} A_{\mathrm{f}} m+\dot{c}_{\mathrm{f}}+\mathrm{i} \Omega c_{\mathrm{f}}+\mathrm{i} \Omega A_{\mathrm{f}} m_{\mathrm{f}}=\frac{\Gamma_{\mathrm{f}}}{\Omega}$

for the liquid outer core, with $\Gamma_{\mathrm{s}}$ being the total torque on the inner core, $\Gamma_{\mathrm{f}}$, the total torque on the outer core.

Those are the Liouville equations for a three-layer Earth as done in Dehant et al. (1993) (here after noted D93) and we have extended these equations in order to account for the effect of a surface fluid layer. Corrections to these equations, as expressed in Greff-Lefftz et al. $(2000,2002)$ are considered as well. We have to consider the effects of the external fluid through the induced changes in three components of the equations: (1) the relative angular momentum; (2) the changes in the inertia tensor; and (3) the interaction torques at the core boundaries.

\subsection{Incremental relative angular momentum}

The relative angular momentum of the external fluid, related to the wind in the atmosphere and to the currents in the ocean, must be included in the angular momentum budget. This is classically done using the motion (or wind) angular momentum excitation function $\left(\chi_{\mathrm{w}}\right)$, as defined in Munk \& McDonald (1960). The wind term of the atmospheric angular momentum is given by

$\boldsymbol{h}_{\mathrm{a}}=\frac{a^{3}}{g} \int_{S} \int\left(\begin{array}{c}v \sin \lambda+u \cos \theta \cos \lambda \\ -v \cos \lambda+u \cos \theta \sin \lambda \\ -u \sin \theta\end{array}\right) \mathrm{d} p \mathrm{~d} S$

where $\theta$ is the colatitude, $\lambda$ is the longitude, $u$ is the zonal wind, $v$, the meridional wind, $a$ is the Earth mean radius, $g$, the acceleration of gravity, and $\mathrm{d} p$ implies that the vertical integration is done in terms of pressure level. The wind excitation function is defined by its relation to the wind term of the angular momentum:

$h_{\mathrm{a}}=\alpha A \Omega \chi_{\mathrm{w}}$

where $\alpha$ is the Earth dynamical flattening and $A$ is the Earth equatorial moment of inertia.

\subsection{Change in the inertia tensor}

The inertia increments are computed by integration on the deformed volume of the core and of the inner core:

$$
\begin{aligned}
& c_{\mathrm{f}}=-\int_{0}^{2 \pi} \int_{0}^{\pi}\left(\int_{r_{\mathrm{ICB}}}^{r_{\mathrm{CMB}}} r^{4} \rho_{\mathrm{f}}(\cos \lambda+i \sin \lambda) \sin ^{2} \theta \cos \theta \mathrm{d} r\right) \mathrm{d} \theta \mathrm{d} \lambda \\
& c_{\mathrm{S}}=-\int_{0}^{2 \pi} \int_{0}^{\pi}\left(\int_{0}^{r_{\mathrm{ICB}}} r^{4} \rho_{\mathrm{s}}(\cos \lambda+i \sin \lambda) \sin ^{2} \theta \cos \theta \mathrm{d} r\right) \mathrm{d} \theta \mathrm{d} \lambda
\end{aligned}
$$

where $r_{\mathrm{ICB}}$ and $r_{\mathrm{CMB}}$ are the radius of the deformed boundaries of the core (Inner Core Boundary and Core Mantle Boundary, respectively), $\rho_{\mathrm{f}}$ and $\rho_{\mathrm{s}}$ are the density of the fluid core and solid inner core respectively. Using the Love number formalism, we can evaluate the topography of the deformed surface from the forcing potential and pressure (Greff-Lefftz et al. 2000). In our 
notation, the load Love numbers are indicated by a hat (they account for pressure and loading mass potential effects), the pressure ones by a bar, and the potential ones left alone. Note that the Love numbers, by definition, include all the static indirect effects, as for instance the deformation due to the change of pressure associated with the external potential acting on the Earth. They do not, however, include the effects related to the dynamics, as for instance the deformation associated with the rotation of the Earth. For this reason, the centrifugal potential is included in the potential considered, and we have the rotation potential $\pi$. If the dynamics effects were included in the Love number, we would not need to have any other effects considered than the external forcing (external potential $W$ and external pressure $P_{\text {ext }}$ ). We did not consider, in our study, the terms in product of Love numbers, which are of the second order. It is not only because those effects are small, considering the numerical value of the Love number, but also because their numerical values are not very reliable, as they are most certainly much affected by the simple approximation done in this study.

As the surface fluid is included in the "Global Earth", we have to take it into account when computing the global increment of inertia. Additionally, the loading of the surface fluids induces deformations, which changes the inertia of the global Earth $\left(c_{\mathrm{E}}\right)$, the core $\left(c_{\mathrm{f}}\right)$ and the inner core $\left(c_{\mathrm{s}}\right)$. The change of inertia of the whole Earth due to the presence at its surface of a thin layer of fluid of which the surface pressure is $P_{\text {ext }}$ is given by

$c_{\mathrm{a}}=\frac{\Omega a^{4}}{g} \int_{S} P_{\mathrm{ext}}\left(\begin{array}{c}\sin \theta \cos \theta \cos \lambda \\ \sin \theta \cos \theta \sin \lambda \\ \sin ^{2} \theta\end{array}\right)$.

The mass (or pressure) angular momentum excitation function is defined by its relation to the inertia change (see Munk \& McDonald 1960):

$c_{\mathrm{a}}=\alpha A \chi_{\mathrm{P}}$.

After some algebra, done for instance in Sasao \& Wahr (1981) and Dehant et al. (1993), it is eventually possible to get the expression of the inertia change as a function of the rotation variation of the whole planet, of the core, and of the inner core, of the external forcing and surface pressure, as well as of properties of the Earth model.

$$
\begin{aligned}
c_{\mathrm{E}}= & \alpha A \frac{k_{0}^{t}}{\kappa} m+\alpha A \frac{\bar{k}_{1}^{t}+\bar{k}_{2}^{t}}{\kappa} m_{\mathrm{f}}+\alpha A \frac{k_{2}^{t}}{\kappa} m_{\mathrm{s}}-3 \alpha A \frac{k_{0}^{t}}{\kappa} \frac{W}{\Omega^{2} a^{2}}+\alpha A\left(1+\hat{k}_{0}^{t}\right) \chi_{\mathrm{p}}-\mathrm{i} \alpha_{\mathrm{s}} A_{\mathrm{s}}\left(1-\frac{\rho_{\mathrm{f}}}{\rho_{\mathrm{s}}}\right)\left(1+\left(\frac{a}{c}\right)^{3} \hat{k}_{2}^{t}\right) \theta_{\mathrm{s}} \\
c_{\mathrm{f}}= & \frac{q_{0}}{2}\left(\left(A_{\mathrm{f}}+\frac{\rho_{\mathrm{f}}}{\rho_{\mathrm{s}}} A_{\mathrm{s}}\right) h_{0}^{\mathrm{f}}-A_{\mathrm{s}} h_{0}^{\mathrm{s}} \frac{\rho_{\mathrm{f}}}{\rho_{\mathrm{s}}}\right) m+\frac{q_{0}}{2}\left(\left(A_{\mathrm{f}}+\frac{\rho_{\mathrm{f}}}{\rho_{\mathrm{s}}} A_{\mathrm{s}}\right) \bar{h}_{1}^{\mathrm{f}}-A_{\mathrm{s}} \bar{h}_{2}^{\mathrm{s}} \frac{\rho_{\mathrm{f}}}{\rho_{\mathrm{s}}}\right) m_{\mathrm{f}} \\
& -A_{\mathrm{s}} h_{\mathrm{s}}^{2} \frac{q_{0}}{2} \frac{\rho_{\mathrm{f}}}{\rho_{\mathrm{s}}} m_{\mathrm{s}}-3\left(\left(A_{\mathrm{f}}+\frac{\rho_{\mathrm{f}}}{\rho_{\mathrm{s}}} A_{\mathrm{s}}\right) h_{0}^{\mathrm{f}}-A_{\mathrm{s}} h_{0}^{\mathrm{s}} \frac{\rho_{\mathrm{f}}}{\rho_{\mathrm{s}}}\right) \frac{q_{0}}{2} \frac{W}{\Omega^{2} a^{2}}+\mathrm{i} \alpha_{\mathrm{s}} A_{\mathrm{s}} \frac{\rho_{\mathrm{f}}}{\rho_{\mathrm{s}}}\left(1+x_{\mathrm{s}} h_{2}^{\mathrm{f}}\right) \theta_{\mathrm{s}}+\left(\left(A_{\mathrm{f}}+\frac{\rho_{\mathrm{f}}}{\rho_{\mathrm{s}}} A_{\mathrm{s}}\right) \hat{h}_{0}^{\mathrm{f}}-A_{\mathrm{s}} \hat{h}_{0}^{\mathrm{s}} \frac{\rho_{\mathrm{f}}}{\rho_{\mathrm{s}}}\right) \alpha \chi_{\mathrm{p}} \\
c_{\mathrm{s}}= & A_{\mathrm{s}} h_{0}^{\mathrm{s}} \frac{q_{0}}{2} m+A_{\mathrm{s}} \bar{h}_{2}^{\mathrm{s}} \frac{q_{0}}{2} m_{\mathrm{f}}+A_{\mathrm{s}} h_{2}^{\mathrm{s}} \frac{q_{0}}{2} m_{\mathrm{s}}-3 A_{\mathrm{s}} h_{0}^{\mathrm{s}} \frac{q_{0}}{2} \frac{W}{\Omega^{2} a^{2}}-\mathrm{i} \alpha_{\mathrm{s}} A_{\mathrm{s}} \theta_{\mathrm{s}}\left(1+x^{\mathrm{s}} \hat{h}_{2}^{\mathrm{s}}\right)+A_{\mathrm{s}} \hat{h}_{0}^{\mathrm{s}} \alpha \chi_{\mathrm{p}}
\end{aligned}
$$

where $\frac{q_{0}}{2}$ is the ratio between the centrifugal and gravitational acceleration at the equator, $\kappa=3(C-A) G /\left(\Omega^{2} r_{\text {surf }}^{5}\right)$ is the fluid Love number. $\Delta \rho=\left(1-\frac{\rho_{\mathrm{f}}}{\rho_{\mathrm{s}}}\right)$. The Love numbers $\left(k_{0}^{t}\right.$ and $\left.h_{0}^{t}\right)$ are the classical Love numbers, expressing the deformation of the Earth in response to a potential; when they are overlined, they express the deformation in response to a pressure. The same numbers, when they have a hat, express the response to a surface load (pressure + gravitational attraction). The same quantities, for the inner core and the outer core, are written with s and f instead of $t$, respectively. The same numbers, with ${ }_{2}$ or $_{1}$ instead of $_{0}$, have the same meaning, but the forcing is applied at the Inner Core Boundary (ICB) or Core Mantle Boundary (CMB) respectively. $\rho, \rho_{\mathrm{f}}, \rho_{\mathrm{s}}$ are the (mean) densities of the whole Earth, of the outer core and of the inner core respectively. As we said in Sect. 2, we allow a tilt of the inner core with respect to the mantle. The angle of this tilt is given by $\theta_{\mathrm{s}}$. This quantity is linked to the inner core wobble by $m_{\mathrm{s}}=\dot{\theta}_{\mathrm{s}}$. For the effect of a load at the ICB on the core inertia, we need to account for the effect of both the deformation of the ICB and the CMB. After some algebra, it can be shown that it is equivalent to have a Love number $h_{2}^{\prime \text { f }}$ equal to

$\frac{\rho_{\mathrm{s}}}{\rho_{\mathrm{f}}} \hat{h}_{2}^{\mathrm{f}}+\frac{A_{\mathrm{s}}}{A_{\mathrm{f}}} \hat{h}_{2}^{\mathrm{s}} \frac{c}{b}$

Additionally, $x_{\mathrm{s}}=(4 \pi G / 5) \Delta \rho c / g, A_{\mathrm{f}}$ and $A_{\mathrm{s}}$ are the equatorial moments of inertia of the core and inner core respectively, $a, b$, and $c$ are the radii of the total Earth, the outer core and the inner core respectively.

\subsection{Change in the interaction torques}

As the atmosphere is part of the global Earth, there is no need to add an atmospheric torque in the equations. Note that this is different from what was done in D93, where the atmospheric pressure was considered as an external forcing. On the other hand, the torques at the core boundaries need to be recomputed in this frame. The angular momentum of the fluid core can be written:

$\frac{\mathrm{d} \boldsymbol{H}_{\mathrm{f}}}{\mathrm{d} t}=\int_{V o c} \boldsymbol{r} \wedge \boldsymbol{\nabla} P_{\mathrm{f}} \mathrm{d} V+\int_{V o c} \boldsymbol{r} \wedge \rho_{\mathrm{f}} \boldsymbol{\nabla} \phi \mathrm{d} V+\int_{V o c} \boldsymbol{r} \wedge \rho_{\mathrm{f}} \boldsymbol{\nabla} W \mathrm{~d} V$. 
Where $V$ oc means the volume of the outer core, where $\boldsymbol{H}_{\mathrm{f}}$ is the core angular momentum, $P_{\mathrm{f}}$ is the fluid pressure, $\phi$ is the gravitational potential associated with the mass redistribution inside the Earth, and $W$ is the forcing tidal potential. Without a fully dynamically consistent model of the core, it is impossible to know the pressure $\left(P_{\mathrm{f}}\right)$ inside the core. Consequently, we use the momentum budget equation to evaluate the pressure: as only conservative forces are acting on the fluid and as its density is homogeneous, its acceleration derives from a potential, and we have

$\boldsymbol{\nabla}(\pi+\psi)=\frac{1}{\rho_{\mathrm{f}}} \boldsymbol{\nabla} P_{\mathrm{f}}+\boldsymbol{\nabla}(W+\phi)$

Where $\psi$ is the centrifugal potential and $\pi$ is the potential associated with the acceleration in the rotating frame. Isolating $P_{\mathrm{f}}$ and substituting in the Eq. (33), we get

$\frac{\mathrm{d} \boldsymbol{H}_{\mathrm{f}}}{\mathrm{d} t}=\int_{V \mathrm{oc}} \boldsymbol{r} \wedge \rho_{\mathrm{f}} \boldsymbol{\nabla}(\pi+\psi) \mathrm{d} V$

As $\pi$ and $\psi$ only depend on the rotation of the core and Earth respectively, they can be written in terms of the unknows of our problem. Nevertheless, the presence of a surface fluid does not change the expression of those potentials in terms of the unknowns. Consequently, the surface fluid will not affect the expression of the total torque acting on the fluid core, but it will affect the values of the potential involved in their expressions.

The torque on the inner core is computed from the same Eq. (33), with the density $\rho_{\mathrm{s}}$ rather than $\rho_{\mathrm{f}}$ for the potential parts. When substituting the expression for the fluid pressure, we get:

$\frac{\mathrm{d} \boldsymbol{H}_{\mathrm{s}}}{\mathrm{d} t}=\int_{V \mathrm{ic}} \boldsymbol{r} \wedge \rho_{\mathrm{f}} \boldsymbol{\nabla}(\pi+\psi-\phi-W) \mathrm{d} V+\int_{V \mathrm{ic}} \boldsymbol{r} \wedge \rho_{\mathrm{s}} \boldsymbol{\nabla} \phi \mathrm{d} V+\int_{V \mathrm{ic}} \boldsymbol{r} \wedge \rho_{\mathrm{s}} \boldsymbol{\nabla} W \mathrm{~d} V$

where $V$ ic means the volume of the inner core. Regrouping the terms in $\phi$ and $W$, we have

$\frac{\mathrm{d} \boldsymbol{H}_{\mathrm{s}}}{\mathrm{d} t}=\int_{V \mathrm{ic}} \boldsymbol{r} \wedge \rho_{\mathrm{f}} \boldsymbol{\nabla}(\pi+\psi) \mathrm{d} V+\int_{V \mathrm{ic}} \boldsymbol{r} \wedge\left(\rho_{\mathrm{s}}-\rho_{\mathrm{f}}\right) \boldsymbol{\nabla}(\phi+W) \mathrm{d} V$.

As in the case of the core, the first term is not affected by the presence of a surface fluid. On the contrary, the second term needs to be modified: the mass of the surface fluid has to be included in the forcing potential, and the resulting deformation has to be included in the mass redistribution potential. This last effect is at the second order, and can thus be neglected. After some (heavy) algebra, we obtain the following final expression for the torque over the inner core:

$$
\begin{aligned}
\Gamma_{\mathrm{s}}= & -3 \mathrm{i} A_{\mathrm{s}}\left(\alpha_{\mathrm{s}}\left(1+k_{0}^{\mathrm{s}}\right)\left(1-\frac{\rho_{\mathrm{f}}}{\rho_{\mathrm{s}}}\right)+\frac{q_{0}}{2} h_{0}^{\mathrm{s}} \frac{\rho_{\mathrm{f}}}{\rho_{\mathrm{s}}}\right) \frac{W}{a^{2}}-\mathrm{i} A_{\mathrm{s}} \Omega^{2} m\left(\alpha_{\mathrm{s}}-\frac{q_{0}}{2} h_{0}^{\mathrm{s}}\right)\left(\frac{\rho_{\mathrm{f}}}{\rho_{\mathrm{s}}}\left(1+k_{2}^{\mathrm{s}}\right)-k_{2}^{\mathrm{s}}\right) \\
& +\mathrm{i} A_{\mathrm{s}} \Omega^{2} m_{\mathrm{f}}\left(\frac{\rho_{\mathrm{f}}}{\rho_{\mathrm{s}}} \frac{q_{0}}{2} \bar{h}_{2}^{\mathrm{s}}-\alpha_{\mathrm{s}}\left(\frac{\rho_{\mathrm{f}}}{\rho_{\mathrm{s}}}\left(1+\bar{k}_{2}^{\mathrm{s}}\right)-\bar{k}_{2}^{\mathrm{s}}\right)\right)+\mathrm{i} A_{\mathrm{s}} \Omega^{2} m_{\mathrm{s}}\left(\frac{\rho_{\mathrm{f}}}{\rho_{\mathrm{s}}} \frac{q_{0}}{2} h_{2}^{\mathrm{s}}-\alpha_{\mathrm{s}}\left(1-\frac{\rho_{\mathrm{f}}}{\rho_{\mathrm{s}}}\right) k_{2}^{\mathrm{s}}\right) \\
& -\frac{5}{3} \Omega^{2}\left(1-\frac{\rho_{\mathrm{f}}}{\rho_{\mathrm{s}}}\right) \alpha_{\mathrm{s}} A_{\mathrm{s}}\left(1+\hat{k}_{0}^{\mathrm{s}}\right) \kappa \chi_{\mathrm{p}} .
\end{aligned}
$$

\subsection{The modified angular momentum budget equations}

Substituting the expression of the increments of inertia and of the interaction torque, it is straightforward to express the angular momentum budget equation in terms of the rotation vector of the mantle, of the core, and of the inner core, and in terms of the forcing potential $(W)$ and the atmospheric excitation functions $\left(\chi_{\mathrm{p}}\right.$ and $\left.\chi_{\mathrm{w}}\right)$.

Using a Fourier transform to express the budget equations at a given frequency. The final form of the equation for the global Earth is:

$$
\begin{aligned}
& \mathrm{i} \Omega m\left(\sigma-\Omega \alpha+\alpha \frac{k_{0}^{t}}{\kappa}(\Omega+\sigma)\right)+\mathrm{i} \Omega m_{\mathrm{f}}(\Omega+\sigma)\left(\frac{A_{\mathrm{f}}}{A}+\alpha\left(\frac{\bar{k}_{2}^{t}}{\kappa}+\frac{\bar{k}_{1}^{t}}{\kappa}\right)\right) \\
& +\mathrm{i} \Omega m_{\mathrm{s}}(\Omega+\sigma)\left(\frac{A_{\mathrm{s}}}{A}+\alpha \frac{k_{2}^{t}}{\kappa}\right)+\alpha_{\mathrm{s}} \Omega \frac{A_{\mathrm{s}}}{A} \theta_{\mathrm{s}}(\Omega+\sigma)\left(1-\frac{\rho_{\mathrm{f}}}{\rho_{\mathrm{s}}}\right)\left(1+\frac{a^{3}}{c^{3}} \hat{k}_{2}^{t}\right)= \\
& -\mathrm{i} \alpha \Omega(\Omega+\sigma)\left(\chi_{\mathrm{p}}\left(1+\hat{k}_{0}^{t}\right)+\chi_{\mathrm{w}}\right)-3 \mathrm{i} \alpha\left(\Omega-(\Omega+\sigma) \frac{k_{0}^{t}}{\kappa}\right) \frac{W}{a^{2} \Omega} .
\end{aligned}
$$


Similarly, the final form of the equation for the fluid core is:

$$
\begin{aligned}
\mathrm{i} \sigma \Omega m+\mathrm{i} \Omega \sigma m \frac{q_{0}}{2}\left(h_{0}^{\mathrm{f}}+\frac{A_{\mathrm{s}}}{A_{\mathrm{f}}} \frac{\rho_{\mathrm{f}}}{\rho_{\mathrm{s}}}\left(h_{0}^{\mathrm{f}}-h_{0}^{\mathrm{s}}\right)\right)+\mathrm{i} \Omega m_{\mathrm{f}}\left(\Omega \alpha_{\mathrm{f}}+(\Omega+\sigma)\right) \\
+\mathrm{i} \Omega \sigma m_{\mathrm{f}} \frac{q_{0}}{2}\left(\bar{h}_{1}^{\mathrm{f}}+\frac{A_{\mathrm{s}}}{A_{\mathrm{f}}} \frac{\rho_{\mathrm{f}}}{\rho_{\mathrm{s}}}\left(\bar{h}_{1}^{\mathrm{f}}-\bar{h}_{2}^{\mathrm{s}}\right)\right)-\mathrm{i} \Omega \sigma m_{\mathrm{s}} \frac{A_{\mathrm{s}}}{A_{\mathrm{f}}} \frac{\rho_{\mathrm{f}}}{\rho_{\mathrm{s}}} \frac{q_{0}}{2} h_{2}^{\mathrm{s}}-\alpha_{\mathrm{s}} \Omega \sigma \theta_{\mathrm{s}} \frac{\rho_{\mathrm{f}}}{\rho_{\mathrm{s}}} \frac{A_{\mathrm{s}}}{A_{\mathrm{f}}}\left(1+x^{\mathrm{s}} h_{2}^{\prime \mathrm{f}}\right)= \\
\quad 3 \mathrm{i} \frac{q_{0}}{2} \sigma\left(h_{0}^{\mathrm{f}}+\frac{A_{\mathrm{s}}}{A_{\mathrm{f}}} \frac{\rho_{\mathrm{f}}}{\rho_{\mathrm{s}}}\left(h_{0}^{\mathrm{f}}-h_{0}^{\mathrm{s}}\right)\right) \frac{W}{\Omega a^{2}}-\mathrm{i} \alpha \Omega \sigma \chi_{\mathrm{p}}\left(\hat{h}_{0}^{\mathrm{f}}+\frac{A_{\mathrm{s}}}{A_{\mathrm{f}}} \frac{\rho_{\mathrm{f}}}{\rho_{\mathrm{s}}}\left(\hat{h}_{0}^{\mathrm{f}}-\hat{h}_{0}^{\mathrm{s}}\right)\right) .
\end{aligned}
$$

And we obtain, for the inner core:

$$
\begin{aligned}
& \mathrm{i} \Omega m\left(\sigma-\Omega \alpha_{\mathrm{s}}\left(1-\frac{\rho_{\mathrm{f}}}{\rho_{\mathrm{s}}}\right)\right)+\mathrm{i} \Omega m \frac{q_{0}}{2} h_{0}^{\mathrm{s}}\left(\sigma+\Omega\left(1-\frac{\rho_{\mathrm{f}}}{\rho_{\mathrm{s}}}\right)\right)-\mathrm{i} \alpha_{\mathrm{s}} \Omega^{2} m\left(1-\frac{\rho_{\mathrm{f}}}{\rho_{\mathrm{s}}}\right) k_{2}^{\mathrm{s}}+\mathrm{i} \alpha_{\mathrm{s}} \Omega^{2} m_{\mathrm{f}} \frac{\rho_{\mathrm{f}}}{\rho_{\mathrm{s}}}+\mathrm{i} \Omega m_{\mathrm{f}} \frac{q_{0}}{2} \bar{h}_{2}^{\mathrm{s}}\left(\sigma+\Omega\left(1-\frac{\rho_{\mathrm{f}}}{\rho_{\mathrm{s}}}\right)\right) \\
& \quad-\mathrm{i} \alpha_{\mathrm{s}} \Omega^{2} m_{\mathrm{f}}\left(1-\frac{\rho_{\mathrm{f}}}{\rho_{\mathrm{s}}}\right) \bar{k}_{2}^{\mathrm{s}}+\mathrm{i} \Omega(\sigma+\Omega) m_{\mathrm{s}}+\mathrm{i} \Omega m_{\mathrm{s}} \frac{q_{0}}{2} h_{2}^{\mathrm{s}}\left(\sigma+\Omega\left(1-\frac{\rho_{\mathrm{f}}}{\rho_{\mathrm{s}}}\right)\right)-\mathrm{i} \alpha_{\mathrm{s}} \Omega^{2} m_{\mathrm{s}}\left(1-\frac{\rho_{\mathrm{f}}}{\rho_{\mathrm{s}}}\right) k_{2}^{\mathrm{s}} \\
& \quad+\alpha_{\mathrm{s}} \Omega \theta_{\mathrm{s}}\left(\sigma+\Omega\left(1-\frac{\rho_{\mathrm{f}}}{\rho_{\mathrm{s}}}\right)\left(1+x^{\mathrm{s}} \hat{h}_{2}^{\mathrm{s}}\right)\right)+\alpha_{\mathrm{s}} \Omega^{2} k_{2}^{\mathrm{s}} \theta_{\mathrm{s}}\left(1-\frac{\rho_{\mathrm{f}}}{\rho_{\mathrm{s}}}\right)=-\mathrm{i} \alpha \Omega \chi_{\mathrm{p}} \bar{h}_{0}^{\mathrm{s}}\left(\sigma+\Omega\left(1-\frac{\rho_{\mathrm{f}}}{\rho_{\mathrm{s}}}\right)\right) \\
& \quad-3 \mathrm{i} \frac{W}{a^{2}}\left(\left(1-\frac{\rho_{\mathrm{f}}}{\rho_{\mathrm{s}}}\right) \alpha_{\mathrm{s}}\left(1+k_{0}^{\mathrm{s}}\right)-\frac{q_{0}}{2 \Omega} h_{0}^{\mathrm{s}}\left(\sigma+\Omega\left(1-\frac{\rho_{\mathrm{f}}}{\rho_{\mathrm{s}}}\right)\right)\right) .
\end{aligned}
$$

Note that the torques computed here are an improved version of those computed in Dehant et al. (1993); the pressure is computed on the deformed surface, which adds a term due to the inner core tilt. We have also considered the deformation effects induced by gravitational interaction with a tilted inner-core as proposed by Greff-Lefftz et al. (2000). Nevertheless, we decided to neglect the terms involving a coupling between deformations. This results in a quite different expression of the pressure effect, and in an additional term in the global Earth budget equation, with respect to D93.

At the one-layer Earth limit, the global Earth equation is equivalent to the ones from Barnes et al. (1983). At the two-layer Earth limit, our expression are equivalent to those of Sasao \& Wahr (1981).

\section{Normal modes and transfer function}

In order to evaluate the normal modes of the system formed by Eqs. (39)-(41) and $\dot{\theta}_{\mathrm{s}}=m_{\mathrm{s}}$, we write the left hand side of those equations in a matrix form (which multiply the vector $\left(m, m_{\mathrm{f}}, m_{\mathrm{s}}, \theta_{\mathrm{s}}\right)^{\mathrm{T}}$.

$\left(\begin{array}{cccc}\sigma\left(1+\epsilon_{11}\right)+\Omega \epsilon_{11}^{\prime} & \left(\frac{A_{\mathrm{f}}}{A}+\epsilon_{12}\right)(\sigma+\Omega) & \left(\frac{A_{\mathrm{s}}}{A}+\epsilon_{13}\right)(\sigma+\Omega) & \mathrm{i} \frac{A_{\mathrm{s}}}{A} \epsilon_{14}(\sigma+\Omega) \\ \sigma\left(1+\epsilon_{21}+\frac{A_{\mathrm{s}}}{A_{\mathrm{f}}} \epsilon_{21}^{\prime}\right) & \sigma\left(1+\epsilon_{22}+\frac{A_{\mathrm{s}}}{A_{\mathrm{f}}} \epsilon_{22}^{\prime}\right)+\Omega\left(1+\epsilon_{22}^{\prime \prime}\right) & \sigma \frac{A_{\mathrm{s}}}{A_{\mathrm{f}}} \epsilon_{23} & \mathrm{i} \sigma \frac{A_{\mathrm{s}}}{A_{\mathrm{f}}} \epsilon_{24} \\ \sigma\left(1+\epsilon_{31}\right)+\Omega \epsilon_{31}^{\prime} & \sigma \epsilon_{32}+\Omega \epsilon_{32}^{\prime} & \sigma\left(1+\epsilon_{33}\right)+\Omega\left(1+\epsilon_{33}^{\prime}\right) & \mathrm{i} \sigma \epsilon_{34}+\mathrm{i} \Omega \epsilon_{34}^{\prime} \\ 0 & 0 & 1 & -\mathrm{i} \frac{\sigma}{\Omega}\end{array}\right)$

with:

$$
\begin{aligned}
& \epsilon_{11}=\alpha \frac{k_{0}^{t}}{\kappa} \quad \epsilon_{11}^{\prime}=-\alpha\left(1-\frac{k_{0}^{t}}{\kappa}\right) \\
& \epsilon_{12}=\alpha\left(\frac{\bar{k}_{1}^{t}}{\kappa}+\frac{\bar{k}_{2}^{t}}{\kappa}\right) \\
& \epsilon_{13}=\alpha \frac{k_{2}^{t}}{\kappa}
\end{aligned}
$$

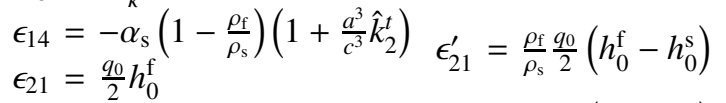

$$
\begin{aligned}
& \epsilon_{22}=\frac{q_{0}}{2} \bar{h}_{1}^{\mathrm{f}} \\
& \epsilon_{23}=-\frac{q_{0}}{2} \frac{\rho_{\mathrm{f}}}{\rho_{\mathrm{s}}} h_{2}^{\mathrm{s}} \\
& \epsilon_{24}=\frac{\rho_{\mathrm{f}}}{\rho_{\mathrm{s}}} \alpha_{\mathrm{s}}\left(1+x^{\mathrm{s}} h_{2}^{\prime \mathrm{f}}\right) \\
& \epsilon_{31}=\frac{q_{0}}{2} h_{0}^{\mathrm{s}} \\
& \epsilon_{32}=\frac{q_{0}}{2} \bar{h}_{2}^{\mathrm{s}} \\
& \epsilon_{33}=\frac{q_{0}}{2} h_{2}^{\mathrm{s}} \\
& \epsilon_{34}=-\alpha_{\mathrm{s}} \\
& \epsilon_{22}^{\prime}=\frac{\rho_{\mathrm{f}}}{\rho_{\mathrm{s}}} \frac{q_{0}}{2}\left(\bar{h}_{1}^{\mathrm{f}}-\bar{h}_{2}^{\mathrm{s}}\right) \\
& \epsilon_{22}^{\prime \prime}=\alpha_{\mathrm{f}}
\end{aligned}
$$

Solving the homogeneous system, we found the frequency of the free modes. For computational reasons, it is easier to separate our problem based on the period: we study separately the low frequency part of the spectrum (for which $\sigma$ is of the order of $\epsilon \Omega$ ), and the quasi-diurnal band (for which $\sigma=-\Omega(1+x$ ), with $x$ of the order of $\epsilon$ ). 
The low frequency case is discussed in Dehant et al. (2005), in the case of Mars. In the nearly diurnal approximation, the matrix is:

$\left(\begin{array}{ccc}-1 & -\frac{A_{\mathrm{f}}}{A} x & \frac{A_{\mathrm{s}}}{A} x \\ -1 & \epsilon_{22}^{\prime \prime}-\epsilon_{22}-\frac{A_{\mathrm{s}}}{A_{\mathrm{f}}} \epsilon_{22}^{\prime}-x & \frac{A_{\mathrm{s}}}{A_{\mathrm{f}}}\left(\epsilon_{23}-\epsilon_{24}\right) \\ -1 & \epsilon_{32}^{\prime}-\epsilon_{32} & x+\left(\epsilon_{33}-\epsilon_{33}^{\prime}-\epsilon_{34}+\epsilon_{34}^{\prime}\right)\end{array}\right)$.

Again, as in D93, we find two quasi-diurnal modes:

$x_{\mathrm{FICN}}=\tilde{\lambda}_{4}\left(1-\frac{A_{\mathrm{s}}}{A_{m}\left(\tilde{\lambda}_{3}-\tilde{\lambda}_{4}\right)}\left(\tilde{\lambda}_{3} \frac{A_{\mathrm{f}}}{A}+\lambda_{y}+\lambda_{z}\right)\right)-\frac{A_{\mathrm{s}}}{A_{\mathrm{f}}} \frac{A}{A_{m}} \frac{\lambda_{y} \lambda_{z}}{\tilde{\lambda}_{3}-\tilde{\lambda}_{4}}$
$x_{\mathrm{FCN}}=\tilde{\lambda}_{3}\left(1+\frac{A_{\mathrm{s}}}{A_{m}\left(\tilde{\lambda}_{3}-\tilde{\lambda}_{4}\right)}\left(\tilde{\lambda}_{4} \frac{A_{\mathrm{f}}}{A}+\lambda_{y}+\lambda_{z}\right)\right)+\frac{A_{\mathrm{s}}}{A_{\mathrm{f}}} \frac{A}{A_{m}} \frac{\lambda_{y} \lambda_{z}}{\tilde{\lambda}_{3}-\tilde{\lambda}_{4}}$

with

$\lambda_{3}=\epsilon_{22}^{\prime \prime}-\epsilon_{22}-\frac{A_{\mathrm{s}}}{A_{\mathrm{f}}} \epsilon_{22}^{\prime}$

$\tilde{\lambda}_{3}=\frac{A-A_{\mathrm{s}}}{A_{m}} \lambda_{3}$

$\lambda_{4}=\epsilon_{33}^{\prime}-\epsilon_{33}-\epsilon_{34}^{\prime}+\epsilon_{34}$

$\tilde{\lambda}_{4}=\frac{A-A_{\mathrm{f}}}{A_{m}} \lambda_{4}$

$\lambda_{y}=\epsilon_{32}^{\prime}-\epsilon_{32}$

$\lambda_{z}=\epsilon_{24}-\epsilon_{23}$.

\subsection{Transfer function for the quasi-diurnal frequency band}

The dynamic equation system is solved in the quasi-diurnal frequency band. After some (heavy) algebra, we find a solution that can be written as:

$$
\begin{aligned}
m= & \left(A_{W}^{\mathrm{TI}}+B_{W}^{\mathrm{TI}} A_{\mathrm{s}}\right) \frac{W}{\Omega^{2} a^{2}}+\left(A_{\mathrm{w}}^{\mathrm{TI}}+B_{\mathrm{w}}^{\mathrm{TI}} A_{\mathrm{s}}\right) \chi_{\mathrm{w}}+\left(A_{\mathrm{p}}^{\mathrm{TI}}+B_{\mathrm{p}}^{\mathrm{TI}} A_{\mathrm{s}}\right) \chi_{\mathrm{p}}+\frac{A_{W}^{\mathrm{FCN}}+B_{W}^{\mathrm{FCN}} A_{\mathrm{s}}}{\Omega^{2} a^{2}\left(x-x_{\mathrm{FCN}}\right)}+\frac{A_{\mathrm{w}}^{\mathrm{FCN}}+B_{\mathrm{w}}^{\mathrm{FCN}} A_{\mathrm{s}}}{x-x_{\mathrm{FCN}}} \chi_{\mathrm{w}} \\
& +\frac{A_{\mathrm{p}}^{\mathrm{FCN}}+B_{\mathrm{p}}^{\mathrm{FCN}} A_{\mathrm{s}}}{x-x_{\mathrm{FCN}}} \chi_{\mathrm{p}}+\frac{A_{W}^{\mathrm{FICN}}+B_{W}^{\mathrm{FICN}} A_{\mathrm{s}}}{\Omega^{2} a^{2}\left(x-x_{\mathrm{FICN}}\right)} W+\frac{A_{\mathrm{w}}^{\mathrm{FICN}}+B_{\mathrm{w}}^{\mathrm{FICN}} A_{\mathrm{s}}}{x-x_{\mathrm{FICN}}} \chi_{\mathrm{w}}+\frac{A_{\mathrm{p}}^{\mathrm{FICN}}+B_{\mathrm{p}}^{\mathrm{FICN}} A_{\mathrm{s}}}{x-x_{\mathrm{FICN}}} \chi_{\mathrm{p}} .
\end{aligned}
$$

For the Earth, $A_{\mathrm{s}}$, which represents the inertia of the inner core, is very small, on the order of $10^{-3}$ of the inertia of the mantle. Consequently, the terms in $A_{\mathrm{s}}$ are usually negligible. Nevertheless, the resonance at the Free Inner Core Nutation makes it possible to enhance terms in $A_{\mathrm{s}}$, which need then to be kept in the equation. Thus, the solution that we use in the following can be expressed as

$$
\begin{aligned}
m= & A_{W}^{\mathrm{TI}} \frac{W}{\Omega^{2} a^{2}}+A_{\mathrm{w}}^{\mathrm{TI}} \chi_{\mathrm{w}}+A_{\mathrm{p}}^{\mathrm{TI}} \chi_{\mathrm{p}}+\frac{A_{W}^{\mathrm{FCN}}}{\Omega^{2} a^{2}\left(x-x_{\mathrm{FCN}}\right)} W+\frac{A_{\mathrm{w}}^{\mathrm{FCN}}}{x-x_{\mathrm{FCN}}} \chi_{\mathrm{w}}+\frac{A_{\mathrm{p}}^{\mathrm{FCN}}}{x-x_{\mathrm{FCN}}} \chi_{\mathrm{p}} \\
& +\frac{B_{W}^{\mathrm{FICN}}}{\Omega^{2} a^{2}\left(x-x_{\mathrm{FICN}}\right)} W+\frac{B_{\mathrm{w}}^{\mathrm{FICN}}}{x-x_{\mathrm{FICN}}} \chi_{\mathrm{w}}+\frac{B_{\mathrm{p}}^{\mathrm{FICN}}}{x-x_{\mathrm{FICN}}} \chi_{\mathrm{p}} .
\end{aligned}
$$

The expression for the coefficients in the above equation are given in the table below. In all the expressions, we limit ourselves to the lowest possible order in the small quantities, for each part of the formula, in order to keep all the physics and the expressions as simple as possible. In particular, this implies that the non-deformation parts of the atmospheric pressure $\left(\chi_{\mathrm{p}}\right)$ terms disappear from the expressions.

\section{Love number for a three-layer Earth}

In order to evaluate the Love numbers, we need to solve the gravito-elasticity equations (as described for instance by Alterman et al. 1959) for a planet composed of three homogeneous layers, using boundary conditions inside the planet and at the surface. Those equations are motion equations, stress-strain relationship, and Poisson equation for the mass redistribution, and the boundary conditions are continuity of the potential, stress, and radial displacement at all the boundaries, and free slip conditions (and thus no tangential stress) at the core boundaries, under the condition that the solution does not diverge at the center of the Earth. 
Table 2. Analytical expression of the resonance strengths in Formula (53). The first column indicates the cause and the normalization of the forcing.

\begin{tabular}{llll}
\hline \hline & $A^{\mathrm{TI}}$ & $A^{\mathrm{FCN}}$ & $B^{\mathrm{FICN}}$ \\
\hline$\frac{3 W}{\Omega^{2} a^{2}}$ & $\alpha \frac{A}{A_{m}}-\frac{q_{0}}{2} h_{\mathrm{f}}^{0} \frac{A_{\mathrm{f}}}{A_{m}}$ & $\frac{\left(\alpha-\frac{q_{0}}{2} h_{\mathrm{f}}^{0}\right) x_{\mathrm{FCN}} A_{\mathrm{f}}}{A_{m}}$ & $\frac{\left(\alpha_{\mathrm{s}}\left(1+k_{0}^{\mathrm{s}}\right) \frac{\rho_{\mathrm{s}}-\rho_{\mathrm{f}}}{\rho_{\mathrm{s}}}+\frac{\rho_{\mathrm{f}}}{\rho_{\mathrm{s}}} \frac{q_{0}}{2} h_{\mathrm{s}}^{0}\right) A_{\mathrm{s}} x_{\mathrm{FICN}}\left(A x_{\mathrm{FICN}}+A \lambda_{z}-A_{m} x_{\mathrm{FCN}}\right)}{A A_{m}\left(x_{\mathrm{FCN}}-x_{\mathrm{FICN}}\right)}$ \\
$\chi_{\mathrm{w}}$ & $-\alpha \frac{A_{\mathrm{f}} x_{\mathrm{FCN}}+A x}{A_{m}}$ & $-\alpha x_{\mathrm{FCN}}^{2} \frac{A_{\mathrm{f}}}{A_{m}}$ & $-\frac{\alpha A_{\mathrm{s}} x_{\mathrm{FICN}}^{2}\left(A x_{\mathrm{FICN}}+A \lambda_{y}-A_{m} x_{\mathrm{FCN}}\right)\left(A x_{\mathrm{FICN}}+A \lambda_{z}-A_{m} x_{\mathrm{FCN}}\right)}{A_{m}^{2} A\left(x_{\mathrm{FCN}}-x_{\mathrm{FICN}}\right)^{2}}$ \\
$\chi_{\mathrm{p}}$ & $\alpha \frac{A_{\mathrm{f}}}{A_{m}} \hat{h}_{\mathrm{f}}^{0}$ & $\alpha x_{\mathrm{FCN}} \hat{h}_{\mathrm{f}}^{0} \frac{A_{\mathrm{f}}}{A_{m}}$ & $-\frac{\alpha A_{\mathrm{s}} x_{\mathrm{FICN}}\left(\bar{h}_{\mathrm{s}}^{0} \frac{\rho_{\mathrm{f}}}{\rho_{\mathrm{s}}}-\hat{h}_{\mathrm{f}}^{0} \frac{A_{\mathrm{f}} x_{\mathrm{f} \mathrm{ICN}}+A \lambda_{y}}{x_{\mathrm{FCN}}-x_{\mathrm{FICN}}}\right)\left(A x_{\mathrm{FICN}}+A \lambda_{z}-A_{m} x_{\mathrm{FCN}}\right)}{A A_{m}\left(x_{\mathrm{FCN}}-x_{\mathrm{FICN}}\right)}$ \\
\hline
\end{tabular}

In order to express our solution, we used simple analytical solutions of the radial dependency of our variables which are, as in Alterman et al. (1959):

- $y_{1}$, the radial displacement;

- $y_{2}$, the radial stress;

- $y_{3}$, the tangential displacement;

- $y_{4}$, the tangential stress;

- $y_{5}$, the mass redistribution potential;

- $y_{6}$, involving $y_{1}$ and the derivative of $y_{5}$.

And the analytical solutions used are the following:

At the Earth surface, the boundary conditions are determined from the forcing. There is no condition on the displacement at the Earth surface; the tangential stress is zero, and the radial stress is continuous, i.e. equal to the external pressure. In addition, there is continuity of the potential and of a combination of its radial derivative and the radial displacement, involving the Poisson equation:

$y_{2}(a)=-P_{\mathrm{ext}}-\frac{(2 n+1) \rho_{M}}{3} S_{\mathrm{ext}}$

$y_{4}(a)=T_{\text {ext }}$

$y_{6}(a)+\frac{n+1}{a} y_{5}(a)=(2 n+1) \frac{W}{a}+(2 n+1) \frac{S_{\text {ext }}}{a}$

where $S_{\text {ext }}$ is a load external potential, $P_{\text {ext }}$ an external pressure, $T_{\text {ext }}$ an external tangential stress, and $W$ an external potential.

At the CMB, we have continuity of the radial displacement, but not of the tangential displacement. In order to allow additional displacement due to non static response of the Earth, we add arbitrary constant $\left(K_{1}\right.$ and $\left.K_{3}\right)$ that will be solved for using the complete system. Additionally, we have continuity of the radial stress (i.e. pressure) and the tangential stress acting only on the mantle. We also use the continuity of the potential and its derivative:

$y_{1}^{\text {mantle }}(b)-y_{1}^{\text {core }}(b)-b K_{1}=0$

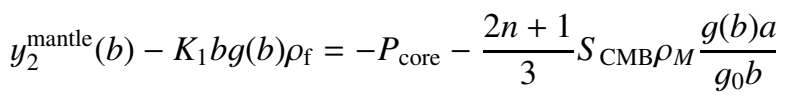

$y_{3}^{\text {mantle }}(b)-b K_{3}=0$

$y_{4}^{\text {mantle }}(b)=-T_{\mathrm{CMB}}$

$y_{5}^{\text {mantle }}(b)-y_{5}^{\mathrm{CMB}}(b)+W_{\mathrm{CMB}}=0$

$y_{6}^{\text {mantle }}(b)-y_{6}^{\text {core }}(b)+3 \frac{b}{a} \frac{\rho_{\mathrm{f}}}{\rho_{M}} K_{1} g_{0}=-\frac{n}{b} W_{\mathrm{CMB}}+\frac{2 n+1}{b} S_{\mathrm{CMB}}$

where $S_{\mathrm{CMB}}$ is a loading potential at the $\mathrm{CMB}, T_{\mathrm{CMB}}$, a tangential stress at the $\mathrm{CMB}$, and $W_{\mathrm{CMB}}$, a potential at the CMB.

At the ICB, the continuity conditions are the same as at the CMB (introducing the arbitrary constants $K_{2}$ and $K_{4}$ ):

$y_{1}^{\text {core }}(c)-y_{1}^{\text {inner core }}(c)-c K_{2}=0$

$-y_{2}^{\text {inner core }}(c)-c K_{2} g(c) \rho_{\mathrm{f}}=P_{\mathrm{ICB}}+\frac{2 n+1}{3} \frac{a}{c} \rho_{M} \frac{g(c)}{g_{0}} S_{\mathrm{ICB}}$

$y_{3}^{\text {inner core }}(c)-c K_{4}=0$

$y_{4}^{\text {inner core }}(c)=T_{\text {ICB }}$ 
$y_{5}^{\text {core }}(c)-y_{5}^{\text {inner core }}(c)-W_{\mathrm{CMB}} \frac{c^{n}}{b^{n}}+W_{\mathrm{ICB}}=0$

$y_{6}^{\text {core }}(c)+3 \frac{c}{a} \frac{\rho_{\mathrm{f}}}{\rho_{M}} K_{2} g_{0}-y_{6}^{\text {inner core }}(c)=\frac{n}{b} \frac{c^{n-1}}{b^{n-1}} W_{\mathrm{CMB}}-\frac{n}{c} W_{\mathrm{ICB}}-\frac{2 n+1}{c} S_{\mathrm{ICB}}$.

Using those equations and boundary conditions, we can solve the system, and we obtain the variables in function of the forcing and the Earth interior properties.

As those equations are all linear in the potential, we easily get the expressions and numerical values of the generalized Love numbers for the three-layer Earth; for instance, the Love number $\bar{h}_{0}^{\mathrm{f}}$ is the numerical coefficient of the $P_{\text {ext }}$ in the expression of the CMB radial deformation $\left(y_{1}^{\text {core }}(b)\right)$. The Love number $h_{0}^{t}$ is, by definition, the value of $y_{1}$ at the surface, when the forcing potential is set to $1 / g_{0}$. Similarly, the other Love numbers $h$ are estimated from the values of $y_{1}$ at the different interfaces, for forcings placed at the relevant interfaces. The values of $k$ are computed as the values of $y_{5}$, when the forcing potential is set to 1 .

Table 4 gives the numerical values of the Earth model properties used in our computation and Table 5 gives the numerical values of the Love numbers obtained in our study. Those values are derived as mean values from PREM (Dziewonski \& Anderson 1981).

\section{Excitation by a white noise}

The solutions for the wobble $m$ as a function of the frequency and the forcing is:

$m=$ independent term $+\frac{a_{\mathrm{FCN}} x_{\mathrm{FCN}} \Omega\left(1+\frac{\sigma_{\mathrm{FCN}}}{\Omega}\right)}{\sigma-\sigma_{\mathrm{FCN}}}+\frac{a_{\mathrm{FICN}} x_{\mathrm{FICN}} \Omega\left(1+\frac{\sigma_{\mathrm{FICN}}}{\Omega}\right)}{\sigma-\sigma_{\mathrm{FICN}}}$.

The polar motion is given by, see for instance Gross (1992),

$p(\sigma)=\frac{1}{1+\frac{\sigma}{\Omega}} m(\sigma)$.

We are looking for an expression of the Power Spectral Density (PSD) of the excited polar motion as a function of the PSD of the atmospheric (or oceanic) excitation. The PSD on the arbitrarily small interval centered at the resonance frequency $\left(\sigma_{\mathrm{R}}\right)$ is computed (Lambert 2003) as

$P(\sigma)=\int_{\sigma_{\mathrm{R}}-\delta \sigma_{\mathrm{R}}}^{\sigma_{\mathrm{R}}+\delta \sigma_{\mathrm{R}}} p(\sigma) p^{*}(\sigma) \mathrm{d} \sigma$

where $\delta \sigma_{\mathrm{R}}$ is the half-size of the window, the ${ }^{*}$ is for complex conjugate, and $p(\sigma)$ is the polar motion excited by the atmosphere at frequency $\sigma$. It is given by

$p(\sigma)=T(\sigma) \chi(\sigma)$,

with $T(\sigma)$, the transfer function as given by Eqs. (69) and (70), and $\chi(\sigma)$ is the excitation function at frequency $\sigma$.

According to our white noise hypothesis, we have

$\chi(\sigma) \chi^{*}(\sigma)=K$

$K$ is independent of the frequency. At frequencies close to the resonance frequency $\sigma_{\mathrm{R}}$, the transfer function is dominated by the term in $1 /\left(\sigma-\sigma_{\mathrm{R}}\right)$. Consequently, in our first order approach, we can limit our-self to that term, and we get, considering Eq. (73),

$P_{\mathrm{R}}=A_{\mathrm{R}}^{2} K \int_{\sigma_{\mathrm{R}}-\delta \sigma_{\mathrm{R}}}^{\sigma_{\mathrm{R}}+\delta \sigma_{\mathrm{R}}}\left|\frac{\sigma_{\mathrm{R}}}{\sigma-\sigma_{\mathrm{R}}}\right|^{2} \mathrm{~d} \sigma$.

Note that, in the nutation case, the frequencies has to be taken in the celestial frame, whereas for the polar motion, they have to be taken in the Earth frame. The resonance frequency is complex and can be written as

$\sigma_{\mathrm{R}}=\sigma_{\mathrm{r}}\left(1-\frac{\mathrm{i}}{2 Q_{\mathrm{R}}}\right)$

where $Q_{\mathrm{R}}$ is the quality factor of the resonance and $\sigma_{\mathrm{r}}$ is the real part of the resonance frequency.

From this expression, it is straightforward to get

$$
\begin{aligned}
P_{\mathrm{R}} & =a_{\mathrm{R}}^{2} K \frac{-\sigma_{\mathrm{r}}}{2 Q_{\mathrm{R}}}\left(1+4 Q_{\mathrm{R}}^{2}\right)\left[\arctan \left(2 Q_{\mathrm{R}}\left(1-\frac{\sigma}{\sigma_{\mathrm{r}}}\right)\right)\right]_{\sigma_{\mathrm{R}}-\delta \sigma_{\mathrm{R}}}^{\sigma_{\mathrm{R}}+\delta \sigma_{\mathrm{R}}} \\
& =4 a_{\mathrm{R}}^{2} K \sigma_{\mathrm{r}} Q_{\mathrm{R}} \arctan \left(\frac{2 Q_{\mathrm{R}}}{\sigma_{\mathrm{r}}} \delta \sigma_{\mathrm{R}}\right) .
\end{aligned}
$$


Table 3. Analytical expression for Alterman et al. (1959) solution of the gravito-elastic deformation equations, generalized for a three-layer Earth.

\begin{tabular}{llll}
\hline \hline & Earth & Core & Inner core \\
\hline$y_{1}$ & $\frac{C_{1}}{r^{n}}+\frac{C_{2}}{r^{n+2}}+C_{3} r^{n+1}+C_{4} r^{n-1}$ & $y_{5} / g_{n}$ & $G_{3} r^{n+1}+G_{4} r^{n-1}$ \\
$y_{2}$ & $\frac{-2 \mu C_{1}\left(n^{2}+3 n-1\right)}{(n+1) r^{n+1}}-\frac{2 C_{2}(n+2) \mu}{r^{n+3}}+\frac{2}{n} C_{3} \mu\left(n^{2}-n-3\right) r^{n}$ & $/$ & $\frac{2}{n} \mu_{g} G_{3}\left(n^{2}-n-3\right) r^{n}+2 \mu_{g} G_{4}(n-1) r^{n-2}$ \\
& $\quad+2 \mu C_{4}(n-1) r^{n-2}-\rho_{m} y_{5}+g \rho_{m} y_{1}$ & & $-\rho_{g} y_{5}+g \rho_{g} y_{1}$ \\
$y_{3}$ & $-\frac{(n-2) C_{1}}{n(n+1) r^{n}}-\frac{C_{2}}{(n+1) r^{n+2}}+\frac{C_{3}(n+3)}{n(n+1)} r^{n+1}+\frac{C_{4}}{n} r^{n-1}$ & $/$ & $G_{3} r^{n+1} \frac{n+3}{n(n+1)}+r^{n-1} \frac{G_{4}}{n}$ \\
$y_{4}$ & $\mu C_{1} \frac{2(n-1)}{n r^{n+1}}+\mu C_{2} \frac{2(n+2)}{(n+1) r^{n+3}}+\mu C_{3} \frac{2(n+2)}{n+1} r^{n}$ & 0 & $2 \mu_{g} G_{3} r^{n} \frac{n+2}{n+1}+2 \mu_{g} G_{4} \frac{n-1}{n} r^{n-2}$ \\
& $+\frac{2(n-1)}{n} \mu C_{4} r^{n-2}$ & & $G_{5} r^{n}$ \\
$y_{5}$ & $C_{5} r^{n}+\frac{C_{6}}{r^{n+1}}$ & $C r^{n}+\frac{D c}{r^{n+1}}$ & $n C r^{n-1}-\frac{D c(n+1)}{r^{n+2}}-\frac{3 g_{0} \rho_{\mathrm{f}} y_{1}}{a \rho_{M}}$ \\
$y_{6}$ & $n C_{5} r^{n-1}-C_{6} \frac{n+1}{r^{n+2}}-\frac{3 g_{0} \rho_{m} y_{1}}{a \rho_{M}}$ & $G_{5} n r^{n-1}-3 \frac{g_{0} \rho_{g} y_{1}}{a \rho_{M}}$ \\
\hline
\end{tabular}

We then choose the $\delta \sigma_{\mathrm{R}}$ so that $99 \%$ of the energy is included in the interval. Imposing the condition gives, for any resonance frequency,

$\delta \sigma_{\mathrm{R}}=31.83 \frac{\sigma_{\mathrm{r}}}{Q_{\mathrm{R}}}$

which leads to

$P=6.22 \chi^{2} a_{\mathrm{R}}^{2} \sigma_{\mathrm{r}} Q_{\mathrm{R}}$

Consequently, if we assume that the forcing PSD is of the same order at the FCN and FICN periods, we can estimate the ratio between the signal at the two resonances:

$\frac{P_{\mathrm{FICN}}^{\text {nutation }}}{P_{\mathrm{FCN}}^{\text {nutation }}}=\frac{a_{\mathrm{FICN}} Q_{\mathrm{FICN}} x_{\mathrm{FICN}}}{a_{\mathrm{FCN}} Q_{\mathrm{FCN}} x_{\mathrm{FCN}}}$

where ${ }^{\text {nutation }}$ indicates that the PSD is for the nutation and not for the associated wobble or the associated polar motion (using Eq. (70) expressed in space).

Using the transfer function of Sect. 4 and the Love numbers of Sect. 5, we can evaluate this ratio for a wind ( $\left.\chi_{\mathrm{w}}\right)$ and a pressure $\left(\chi_{\mathrm{p}}\right)$ excitation. First, the ratio between the resonance amplitude is given by:

$\frac{a_{\mathrm{FICN}}^{\chi_{\mathrm{p}}}}{a_{\mathrm{FCN}}^{\chi_{\mathrm{p}}}}=-1.37 \times 10^{-3}$
$\frac{a_{\mathrm{FICN}}^{\chi_{\mathrm{w}}}}{a_{\mathrm{FCN}}^{\chi_{\mathrm{w}}}}=-1.42 \times 10^{-3}$.

Those numbers are at the same order as what would be obtained, in the case of a forcing by an external potential, from the numbers of Table 3b in Mathews et al. (2002).

Using our expression for the resonance frequency, we obtain a ratio between $x_{\mathrm{FICN}}$ and $x_{\mathrm{FCN}}$ of 1.07 . Note that, by using the adopted values for the periods of these mode (see Mathews et al. 2002), this ratio is slightly larger than 2. Our simplified model does not consider any dissipation, and thus can not provide a ratio between the quality factor. Consequently, we use the ones of Mathews et al. (2002), whose ratio is 0.034 .

The ratio between the atmospherically excited FICN and FCN is thus at the level of $7 \times 10^{-8}$. Using this ratio, if we consider the same excitation of the FICN as of the FCN, we would get an amplitude of $0.001 \mu$ as, if excited by white noise only. Note that the period of the FICN, as expected by Mathews et al. (2002), is close to the ENSO band, which might create a non white noise excitation around the FICN period (2.7 years). In Yseboodt (2002), it was shown that the $S_{1}$ excitation might have been modulated by the ENSO cycle. This might be the only chance to see the atmospherically induced free mode of the FICN, as the level of the observation precision is 0.01 mas.

\section{Conclusions}

In this paper, we investigate the response of a three-layered Earth to an atmospheric or oceanic forcing. In particular, we were interested to evaluate the level at which the FICN could be excited by an atmospheric random signal. This study was motivated 
Table 4. Numerical value of the Earth model properties, from PREM.

\begin{tabular}{rl|lll}
\hline \hline$\rho_{\mathrm{f}}$ & $=10890 \mathrm{~kg} \mathrm{~m}^{-3}$ & $\rho_{\mathrm{s}}$ & $=12890 \mathrm{~kg} \mathrm{~m}^{-3}$ \\
$\rho_{m}$ & $=4450 \mathrm{~kg} \mathrm{~m}^{-3}$ & $\rho_{M}$ & $=5514 \mathrm{~kg} \mathrm{~m}^{-3}$ \\
$\mu_{m}$ & $=1.7610^{11} \mathrm{~Pa}$ & $\mu_{g}$ & $=1.6410^{11} \mathrm{~Pa}$ \\
$A$ & $=8.3810^{37} \mathrm{~kg} \mathrm{~m}^{2}$ & $A_{\mathrm{f}}$ & $=9.2610^{36} \mathrm{~kg} \mathrm{~m}^{2}$ \\
$A_{\mathrm{s}}$ & $=8.8610^{34} \mathrm{~kg} \mathrm{~m}^{2}$ & $A_{m}$ & $=7.3810^{37} \mathrm{~kg} \mathrm{~m}^{2}$ \\
$a$ & $=6371000 \mathrm{~m}$ & $b$ & $=3480000 \mathrm{~m}$ \\
$c$ & $=1221000 \mathrm{~m}$ & $q_{0}$ & $=0.00345$ \\
\hline
\end{tabular}

Table 5. Numerical value of the Love numbers.

\begin{tabular}{ll|lll}
\hline \hline$h_{0}^{t}=0.6116$ & $\hat{k}_{2}^{t}=0.0076$ \\
$h_{0}^{\mathrm{f}}=0.3480$ & $\hat{h}_{0}^{\mathrm{f}}=-0.1470$ \\
$h_{0}^{\mathrm{s}}=0.015$ & $\bar{h}_{1}^{\mathrm{f}}=0.3450$ \\
$\hat{h}_{0}^{\mathrm{s}}=4.8 \times 10^{-6}$ & $\hat{h}_{2}^{\mathrm{f}}=1.34 \times 10^{-4}$ \\
$h_{2}^{\mathrm{s}}=0.0468$ & $\bar{h}_{0}^{\mathrm{s}}=-6.1 \times 10^{-4}$ \\
$\hat{h}_{2}^{\mathrm{s}}=-0.0072$ & $\bar{h}_{2}^{\mathrm{s}}=-0.0395$ \\
$k_{0}^{t}=0.3359$ & $k_{2}^{t}=5.5 \times 10^{-6}$ \\
$k_{0}^{\mathrm{s}}=0.0410$ & $\hat{k}_{0}^{t}=-0.2757$ \\
$\bar{k}_{1}^{t}=0.0355$ & $\bar{k}_{2}^{\mathrm{s}}=-0.0086$ \\
$k_{2}^{\mathrm{s}}=0.0102$ & $\bar{k}_{2}^{\mathrm{t}}=2.38 \times 10^{-6}$ \\
\hline
\end{tabular}

by detection of a prograde signal in the 1990-95 VLBI observations at a period consistent with a possible FICN signal, at the level of $20 \mu \mathrm{as}$. As the effect of the external gravitational forcing was already removed in the residuals, only an atmospheric or oceanic source was possible, if the cause was to be geophysical. In the light of the above results, it is extremely unlikely that the atmosphere might excite such a "large" signal. Consequently, we have to find the explanation of those residuals either in the nutation model or in the observation. In a companion paper, Feissel-Vernier et al. (2005) investigate the mapping of slight instabilities in the VLBI celestial reference frame onto the nutation estimation. They find that the intense activity taking place in the extragalactic radio sources gives rise to a jitter in their observed directions at the level of tens of $\mu$ as. By chance it happened that these apparent motions could mimic a $20 \mu$ as 880-day prograde periodic component in the celestial pole motion over the 1990-95 time span. This seems to be a much more convincing explanation for the observed residuals.

Nevertheless, we can not rule out the (remote) possibility that, in the geophysical fluids (atmosphere, ocean, core), there would be a free mode at a frequency corresponding exactly to that frequency, which would excite the FICN to an observable level. At present, the quality of the diurnal cycle in the Global Circulation Models of those fluids is much too low to really investigate this option. We used a simplified Earth model, which might create some imprecision in our results. Nevertheless, considering the order of magnitude between the observable level and the possible FICN excitation, it is not likely that it would change our conclusion.

Acknowledgements. The work of OdV was financially supported by the Belgian Service Public fédéral de Programmation Politique scientifique. The authors thank C. Bizouard and S. Lambert for useful discussions.

\section{References}

Alterman, Z., Jarosch, H., \& Pekeris, C. L. 1959, Proc. R. Soc. London A, 252, 80

Barnes, R. T. H., Hide, R., White, A. A., \& Wilson, C. A. 1983, Proc. R. Soc. London A, 387, 31

Brzezinski, A. 1994, Manuscripta Geodaetica, 19, 157

Brzezinski, A., Bizouard, C., \& Petrov, S. 2002, Surveys Geophys., 23, 33

Dehant, V., Hinderer, J., Legros, H., \& Lefftz, M. 1993, Phys. Earth Planet. Inter., 76, 259

Dehant, V., Feissel-Vernier, M., de Viron, O., et al. 2003, J. Geophys. Res., 108(B5), 2275

Dehant, V., de Viron, O., Van Hoolst, T., \& Karatekin, O. 2005, in preparation 
Dziewonski, A. D., \& Anderson, D. L. 1981, Phys. Earth Planet. Inter., 25, 297

Feissel-Vernier, M. 2003, A\&A, 403, 105

Feissel-Vernier, M., Ma, C., Gontier, A.-M., \& Barache, C. 2005, A\&A, 438, 1141

Greff-Lefftz, M., Legros, H., \& Dehant, V. 2000, Phys. Earth planet. Inter., 122, 187

Greff-Lefftz, M., Dehant, V., \& Legros, H. 2002, Phys. Earth planet. Inter., 129, 31

Gross, R. S. 1992, Geophys. J. Int., 109, 162

Herring, T. A., Buffett, B. A., Mathews, P. M., \& Shapiro, I. I. 1991, J. Geophys. Res., 96(B5), 8259

Herring, T. A., Mathews, P. M., \& Buffett, B. A. 2002, J. Geophys. Res., 107(B4)

Lambert, S. 2003, Ph.D. Thesis, Observatoire de Paris, France [in French]

Mathews, P. M., Herring, T. A., \& Buffett, B. A. 2002, J. Geophys. Res., 107(B10)

Munk, W. H., \& McDonald, G. F. 1960, The Rotation of the Earth, a Geophysical discussion (Cambridge University Press), 323

Sasao, T., \& Wahr, J. M. 1981, Geophys. J. R. Astron. Soc., 64, 729

Yseboodt, M., de Viron, O., Chin, T. M. , \& Dehant., V. 2002, J. Geophys. Res., 107(B2) 